TRANSACTIONS OF THE

AMERICAN MATHEMATICAL SOCIETY

Volume 363, Number 8, August 2011, Pages 4383-4399

S 0002-9947(2011)05291-8

Article electronically published on March 22, 2011

\title{
ON THE CARTAN MATRIX OF MACKEY ALGEBRAS
}

\author{
SERGE BOUC
}

\begin{abstract}
Let $k$ be a field of characteristic $p>0$, and let $G$ be a finite group. The first result of this paper is an explicit formula for the determinant of the Cartan matrix of the Mackey algebra $\mu_{k}(G)$ of $G$ over $k$. The second one is a formula for the rank of the Cartan matrix of the cohomological Mackey algebra $\operatorname{co\mu }_{k}(G)$ of $G$ over $k$, and a characterization of the groups $G$ for which this matrix is nonsingular. The third result is a generalization of this rank formula and characterization to blocks of $\operatorname{co\mu }_{k}(G)$ : in particular, if $b$ is a block of $k G$, the Cartan matrix of the corresponding block $\operatorname{co\mu }_{k}(b)$ of $\operatorname{co\mu }_{k}(G)$ is nonsingular if and only if $b$ is nilpotent with cyclic defect groups.
\end{abstract}

\section{INTRODUCTION}

1.1. The theory of Mackey functors for a finite group $G$ over a commutative ring $R$ is by many aspects very similar to the theory of $R G$-modules. It has been shown by J. Thévenaz and P. Webb ([13]), among many fundamental other results, that the category of Mackey functors for $G$ over $R$ is equivalent to the category of modules over the Mackey algebra $\mu_{R}(G)$. This algebra shares many properties with the group algebra $R G$ : it is free as an $R$-module, and its $R$-rank does not depend on $R$; if $K$ is a field of characteristic 0 or coprime to the order of $G$, the algebra $\mu_{K}(G)$ is semisimple; when $(K, \mathcal{O}, k)$ is a $p$-modular system, there is a decomposition theory from Mackey functors for $G$ over $K$ to Mackey functors for $G$ over $k$; the Cartan matrix of $\mu_{k}(G)$ is symmetric and nonsingular. This list of common properties between the Mackey algebra and the group algebra is far from exhaustive.

1.2. However, some well-known results for group algebras are no longer true for Mackey algebras. It was observed on small examples, in particular by M. Nicollerat in her thesis ([12] Chapitre 5), that the determinant of the Cartan matrix of $\mu_{k}(G)$, when $k$ is a field of characteristic $p$, is generally not a power of $p$, even when $G$ itself is a $p$-group. Instead, some rather strange prime factors appear in this determinant. One of the motivations of this paper is to give an explanation for these strange factors, by stating an explicit formula for the determinant of the Cartan matrix of $\mu_{k}(G)$ (Theorem 2.28).

1.3. The other motivation is the similar problem for the cohomological Mackey algebra $\operatorname{co\mu }_{k}(G)$, which was defined by Thévenaz and Webb as a specific quotient of $\mu_{k}(G)$, with the property that the modules over $\operatorname{co\mu }_{k}(G)$ are exactly the cohomological Mackey functors: the first major difference is that, in general, the Cartan matrix of this algebra is singular. This raises the question of characterizing those

Received by the editors October 6, 2009 and, in revised form, January 2, 2010.

2010 Mathematics Subject Classification. Primary 18G05, 20C20, 20 J06.

Key words and phrases. Cartan matrix, cohomological, Mackey functor, block.

(c) 2011 American Mathematical Society Reverts to public domain 28 years from publication 4383 
finite groups $G$ for which the Cartan matrix of $\operatorname{co\mu }_{k}(G)$ is nonsingular, and the answer is the second main result of this paper (Theorem 3.3): these groups are exactly the $p$-nilpotent groups with cyclic Sylow $p$-subgroups. The other possibly interesting result is that for an arbitrary finite group $G$, the rank of the Cartan matrix of $\operatorname{co\mu }_{k}(G)$ is equal to the number of conjugacy classes of pairs $(R, s)$, where $R$ is a cyclic $p$-subgroup of $G$, and $s$ is a $p^{\prime}$-element of the centralizer of $R$ in $G$.

The third result of this paper is a natural generalization of Theorem 3.3 to blocks, suggested by the one-to-one correspondence $b \mapsto c o \mu_{k}(b)$ between blocks of $k G$ and blocks of $\operatorname{co\mu }_{k}(G)$. Theorem 4.2 gives a formula for the rank of the Cartan matrix of $\operatorname{co\mu }_{k}(b)$, in terms of $b$-Brauer pairs, and shows that this matrix is nonsingular if and only if the block $b$ is nilpotent with cyclic defect groups.

1.4. The paper is organized according to these results. Section 2 is devoted to the case of $\mu_{k}(G)$, starting by recalling some standard notation, definitions and properties, and Section 3 deals with $\operatorname{co\mu }_{k}(G)$. Finally, Section 4 is devoted to the case of blocks of cohomological Mackey functors.

\section{The Mackey Algebra}

2.1. Throughout the paper when $G$ is a finite group and $p$ is a prime number, the set of $p^{\prime}$-elements of $G$ is denoted by $G_{p^{\prime}}$, and the symbol $\left[G_{p^{\prime}}\right]$ denotes a set of representatives of $G$-conjugacy classes in $G_{p^{\prime}}$. The set of $p$-subgroups of $G$ is denoted by $\mathcal{S}_{p}(G)$, and $\left[\mathcal{S}_{p}(G)\right]$ denotes similarly a set of representatives of $G$ conjugacy classes in $\mathcal{S}_{p}(G)$.

2.2. From now on $G$ will be a fixed finite group, and $p$ a fixed prime number. Let $(K, \mathcal{O}, k)$ be a $p$-modular system. Thus $\mathcal{O}$ is a discrete valuation ring with residue field $k$ of characteristic $p$, and field of fractions $K$ of characteristic 0 . Assume that $K$ and $k$ are splitting fields for all the groups $N_{G}(Q) / Q$, for $Q \in \mathcal{S}_{p}(G)$ (e.g. assume that $K$ contains the $e$-th roots of unity, where $e$ is the exponent of $G$ ).

2.3. When $R$ is a commutative ring with identity element, the Mackey algebra $\mu_{R}(G)$ of $G$ over $R$ has been defined by Thévenaz and Webb ([13], Section 3). It is an associative algebra with the property that the category $\mu_{R}(G)$-Mod of left $\mu_{R}(G)$-modules is equivalent to the category $\operatorname{Mack}_{R}(G)$ of Mackey functors for $G$ over $R$.

2.4. Thévenaz and Webb have also shown (13, Theorem 10.1) that there is an equivalence of abelian categories

$$
\operatorname{Mack}_{k}(G) \cong \prod_{H} \operatorname{Mack}_{k}\left(N_{G}(H) / H, \mathbf{1}\right),
$$

where $H$ runs through a set of representatives of conjugacy classes of $p$-perfect subgroups of $G$, and $\operatorname{Mack}_{k}\left(N_{G}(H) / H, \mathbf{1}\right)$ denotes the subcategory of $\operatorname{Mack}_{k}\left(N_{G}(H) / H\right)$ consisting of Mackey functors which are projective relative to $p$-subgroups. The category $\operatorname{Mack}_{k}(G, \mathbf{1})$ is equivalent to $\mu_{k}(G, \mathbf{1})$-Mod, where $\mu_{k}(G, \mathbf{1})$ is a direct summand of $\mu_{k}(G)$ of the form $\mu_{k}(G) f$, for a specific central idempotent $f$ of $\mu_{k}(G)$.

2.5. (13] Theorem 12.7 and Corollary 12.8) The correspondence $M \mapsto M(\mathbf{1})$, which is evaluation at the trivial subgroup of $G$, induces a one-to-one correspondence between the set of isomorphism classes of indecomposable projective $\mu_{k}(G, \mathbf{1})$ modules and the set of isomorphism classes of indecomposable $p$-permutation $k G$ modules (also called trivial source $k G$-modules). 
2.6. Let $p p_{k}(G)$ denote the Green ring of p-permutation $k G$-modules: as a group, it is the Grothendieck group of the category of finitely generated $p$-permutation $k G$-modules, for relations given by direct sum decompositions. The product on $p p_{k}(G)$ is induced by the tensor product of $k G$-modules over $k$.

If $W$ is a finitely generated $p$-permutation $k G$-module, its dual $W^{*}=\operatorname{Hom}_{k}(W, k)$ is also a $p$-permutation $k G$-module, and this duality extends to a ring automorphism $W \mapsto W^{*}$ of $p p_{k}(G)$.

When $Q$ is a $p$-subgroup of $G$, the Brauer quotient $W[Q]$ is a $p$-permutation $k \bar{N}_{G}(Q)$-module, where $\bar{N}_{G}(Q)=N_{G}(Q) / Q$ (see [7]). This construction commutes with duality and tensor product of $p$-permutation modules: if $V$ and $W$ are (finitely generated) $p$-permutation $k G$-modules, there are isomorphisms of $k N_{G}(Q) / Q$-modules

$$
W[Q]^{*} \cong W^{*}[Q], \quad V[Q] \otimes W[Q] \cong(V \otimes W)[Q] .
$$

In particular, the Brauer quotient induces a ring homomorphism from $p p_{k}(G)$ to $p p_{k}\left(\bar{N}_{G}(Q)\right)$, still denoted by $W \mapsto W[Q]$ (see e.g. [5], Proposition 2.11).

There is a $\mathbb{Z}$-valued bilinear form $\langle$,$\rangle on p p_{k}(G)$ defined for $p$-permutation $k G$-modules $V$ and $W$ by

$$
\langle V, W\rangle_{G}=\operatorname{dim}_{k} \operatorname{Hom}_{k G}(V, W) .
$$

It is worth noticing that

$$
\langle V, W\rangle_{G}=\operatorname{dim}_{k} \operatorname{Hom}_{k G}\left(k, V^{*} \otimes_{k} W\right) .
$$

The bilinear form $\langle,\rangle_{G}$ extends to a $K$-valued bilinear form on $K \otimes_{\mathbb{Z}} p p_{k}(G)$, still denoted by $\langle,\rangle_{G}$.

2.9. Let $\left\langle\langle,\rangle_{G}\right.$ denote the bilinear form on $p p_{k}(G)$ defined for $p$-permutation $k G$-modules $V$ and $W$ by

$$
\begin{aligned}
\langle\langle V, W\rangle\rangle_{G} & =\sum_{Q \in\left[\mathcal{S}_{p}(G)\right]}\langle V[Q], W[Q]\rangle_{\bar{N}_{G}(Q)} \\
& =\sum_{Q \in\left[\mathcal{S}_{p}(G)\right]} \operatorname{dim}_{k} \operatorname{Hom}_{k \bar{N}_{G}(Q)}(V[Q], W[Q]) .
\end{aligned}
$$

It follows from (2.7) that

$$
\langle\langle V, W\rangle\rangle_{G}=\left\langle\left\langle k, V^{*} \otimes_{k} W\right\rangle\right\rangle_{G} .
$$

It was shown in [3], Proposition 5.11 that if $L$ and $M$ are projective Mackey functors in $\operatorname{Mack}_{k}(G, \mathbf{1})$, then

$$
\operatorname{dim}_{k} \operatorname{Hom}_{\operatorname{Mack}_{k}(G, \mathbf{1})}(L, M)=\langle\langle L(\mathbf{1}), M(\mathbf{1})\rangle\rangle_{G} .
$$

When $L$ and $M$ are indecomposable, this is equal to the coefficient $c_{L, M}$ of the Cartan matrix of the algebra $\mu_{k}(G, \mathbf{1})$.

2.11. Let $\mathcal{Q}_{G, p}$ denote the set of pairs $(R, s)$ consisting of a $p$-subgroup $R$ of $G$ and a $p^{\prime}$-element $s$ of $\bar{N}_{G}(R)$. The group $G$ acts by conjugation on $\mathcal{Q}_{G, p}$. Let $\left[\mathcal{Q}_{G, p}\right]$ denote a set of representatives of $G$-orbits on $\mathcal{Q}_{G, p}$, and let $N_{G}(R, s)$ denote the stabilizer of $(R, s) \in \mathcal{Q}_{G, p}$ in $G$ : it is the set of elements $g \in N_{G}(R)$ such that the image $\bar{g}$ of $g$ in $\bar{N}_{G}(R)$ centralizes $s$. In other words, there is an exact sequence of groups

$$
\mathbf{1} \rightarrow R \rightarrow N_{G}(R, s) \rightarrow C_{\bar{N}_{G}(R)}(s) \rightarrow \mathbf{1}
$$


It was shown in [5] that the primitive idempotents of the (commutative) ring $K \otimes_{\mathbb{Z}}$ $p p_{k}(G)$ are indexed by the orbits of $G$ on $\mathcal{Q}_{G, p}$ : the idempotent $F_{R, s}^{G}$ associated to the orbit of the pair $(R, s)$ is equal to

$$
F_{R, s}^{G}=\frac{1}{|R||s|\left|C_{\bar{N}_{G}(R)}(s)\right|} \sum_{\varphi, L} \tilde{\varphi}\left(s^{-1}\right)|L| \mu(L,\langle s R\rangle) \operatorname{Ind}_{L}^{G} \operatorname{Res}_{L}^{\langle s R\rangle} k_{\varphi},
$$

where:

- The group $\langle s R\rangle$ is the inverse image in $N_{G}(R)$ of the subgroup $\langle s\rangle$ of $\bar{N}_{G}(R)$ under the map $x \mapsto x R$.

- The morphism $\varphi$ runs through group homomorphisms $\langle s\rangle \rightarrow k^{\times}$, and $\tilde{\varphi}$ lifts $\varphi$ to $K$. The module $k_{\varphi}$ is the vector space $k$ on which $\langle s R\rangle$ acts by $\langle s R\rangle \rightarrow\langle s\rangle \stackrel{\varphi}{\rightarrow} k^{\times}$(thus $\tilde{\varphi}$ is the Brauer character of the module $k_{\varphi}$ ).

- The group $L$ runs through the set of subgroups of $\langle s R\rangle$ such that $L R=$ $\langle s R\rangle$.

The idempotents $F_{R, s}^{G}$, for $(R, s) \in\left[\mathcal{Q}_{G, p}\right]$, form a $K$-basis of $K \otimes_{\mathbb{Z}} p p_{k}(G)$. Any element $W$ of $K \otimes_{\mathbb{Z}} p p_{k}(G)$ can be expressed in this basis as

$$
W=\sum_{(R, s) \in\left[\mathcal{Q}_{G, p}\right]} F_{R, s}^{G} \otimes_{k} W=\sum_{(R, s) \in\left[\mathcal{Q}_{G, p}\right]} t_{R, s}^{G}(W) F_{R, s}^{G},
$$

where $t_{R, s}^{G}$ is the extension to $K \otimes_{\mathbb{Z}} p p_{k}(G)$ of the species (i.e. the ring homomorphism; see 1, Lemma 2.2.1, page 26) $p p_{k}(G) \rightarrow K$ associated to the pair $(R, s)$.

2.15. Recall that the value of $t_{R, s}^{G}$ on a $p$-permutation $k G$-module $W$ is equal to the value at $s$ of the Brauer character of the Brauer quotient $W[R]$ (see [5, Notation 2.15 and Proposition 2.18). This will also be called the Brauer trace of $s$ on $W[R]$, and denoted by $\operatorname{Br} \operatorname{Tr}(s \mid W[R])$.

2.16. The ring automorphism $W \mapsto W^{*}$ extends by $K$-linearity to an automorphism of the ring $K \otimes_{\mathbb{Z}} p p_{k}(G)$. This automorphism preserves the set of primitive idempotents. The bilinear form $\left\langle\langle,\rangle_{G}\right.$ also extends to a $K$-valued bilinear form on $K \otimes_{\mathbb{Z}} p p_{k}(G)$. Since the dual of $k_{\varphi}$ is isomorphic to $k_{\varphi^{-1}}$, and since $\widetilde{\left(\varphi^{-1}\right)}\left(s^{-1}\right)=\tilde{\varphi}(s)$, it follows from (2.13) that $\left(F_{R, s}^{G}\right)^{*}=F_{R, s^{-1}}^{G}$. Now equation (2.10) shows that

$$
\left\langle\left\langle F_{R^{\prime}, s^{\prime}}^{G}, F_{R, s}^{G}\right\rangle_{G}=\left\{\begin{array}{cl}
\left\langle\left\langle k, F_{R, s}^{G}\right\rangle_{G}\right. & \text { if }\left(R^{\prime}, s^{\prime}\right)==_{G}\left(R, s^{-1}\right) \\
0 & \text { otherwise }
\end{array}\right.\right.
$$

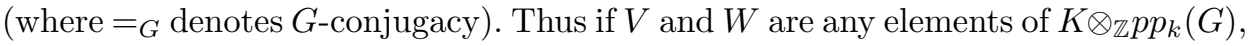
it follows from (2.14) that

$$
\langle\langle V, W\rangle\rangle_{G}=\sum_{(R, s) \in\left[\mathcal{Q}_{G, p}\right]} t_{R, s^{-1}}^{G}(V) t_{R, s}^{G}(W)\left\langle\left\langle k, F_{R, s}^{G}\right\rangle\right\rangle_{G} .
$$

2.17. The indecomposable p-permutation $k G$-modules are indexed by conjugacy classes of pairs $(P, E)$, where $P$ is a $p$-subgroup of $G$, and $E$ is an indecomposable projective $k \bar{N}_{G}(P)$-module $\left(\left[7\right.\right.$, , Theorem 3.2). Let $\left[\mathcal{P}_{G, p}\right]$ denote a set of representatives of such conjugacy classes in $G$.

The indecomposable module $M_{P, E}$ indexed by the pair $(P, E)$ has vertex $P$, and the projective module $E$ is isomorphic to the Brauer quotient $M_{P, E}[P]$. In 
particular, if $Q$ is a subgroup of $G$, then $M_{P, E}[Q]$ is nonzero only if $Q \leq_{G} P$ (and in fact, if and only if $Q \leq_{G} P$ ).

2.18. The modules $M_{P, E}$, for $(P, E) \in\left[\mathcal{P}_{G, p}\right]$, form a basis of $p p_{k}(G)$, hence also a basis of $K \otimes_{\mathbb{Z}} p p_{k}(G)$. The Cartan matrix $\mathrm{C}\left(\mu_{k}(G, 1)\right)$ of the algebra $\mu_{k}(G, \mathbf{1})$ can be viewed as the square matrix indexed by $\left[\mathcal{P}_{G, p}\right]$, where the coefficient indexed by the elements $(P, E)$ and $(Q, F)$ of $\left[\mathcal{P}_{G, p}\right]$ is equal to

$$
\begin{aligned}
c_{(P, E),(Q, F)} & =\left\langle\left\langle M_{P, E}, M_{Q, F}\right\rangle_{G}\right. \\
& =\sum_{(R, s) \in\left[\mathcal{Q}_{G, p}\right]} t_{R, s^{-1}}^{G}\left(M_{P, E}\right) t_{R, s}^{G}\left(M_{Q, F}\right)\left\langle\left\langle k, F_{R, s}^{G}\right\rangle_{G} .\right.
\end{aligned}
$$

2.19. Let $\mathrm{T}$ and $\mathrm{T}^{\prime}$ denote the matrices indexed by the product $\left[\mathcal{P}_{G, p}\right] \times\left[\mathcal{Q}_{G, p}\right]$, defined by

$$
\begin{aligned}
\mathrm{T}_{(P, E),(R, s)} & =t_{R, s}^{G}\left(M_{P, E}\right), \\
\mathrm{T}_{(P, E),(R, s)}^{\prime} & =t_{R, s^{-1}}^{G}\left(M_{P, E}\right),
\end{aligned}
$$

respectively. Note that $\mathrm{T}$ and $\mathrm{T}^{\prime}$ are square matrices. Moreover, let $\mathrm{S}$ be the (square) diagonal matrix indexed by $\left[\mathcal{Q}_{G, p}\right]$, where the diagonal term indexed by $(R, s)$ is equal to $\left\langle\left\langle F_{R, s}^{G}, k\right\rangle\right\rangle_{G}$. It follows that

$$
\mathrm{C}\left(\mu_{k}(G, \mathbf{1})\right)=\mathrm{T}^{\prime} \cdot \mathrm{S} \cdot{ }^{t} \mathrm{~T} .
$$

Thus

$$
\operatorname{det} \mathrm{C}\left(\mu_{k}(G, \mathbf{1})\right)=\operatorname{det} \mathrm{T}^{\prime} \operatorname{det} \mathrm{S} \operatorname{det}^{t} \mathrm{~T},
$$

and moreover, since $\mathrm{S}$ is diagonal,

$$
\operatorname{det} S=\prod_{(R, s) \in\left[\mathcal{Q}_{G, p}\right]}\left\langle\left\langle k, F_{R, s}^{G}\right\rangle\right\rangle_{G}=\prod_{R \in\left[\mathcal{S}_{p}(G)\right]} \prod_{s \in\left[\bar{N}_{G}(R)_{p^{\prime}}\right]}\left\langle\left\langle k, F_{R, s}^{G}\right\rangle\right\rangle_{G} .
$$

2.22. Now since $M_{P, E}[R]=\{0\}$ unless $R \leq_{G} P$, the matrices $\mathrm{T}$ and $\mathrm{T}^{\prime}$ are actually block triangular, with diagonal blocks $\Delta(P)$ indexed by the conjugacy classes of $p$-subgroups $P$ of $G$. Thus

$$
\operatorname{det} \mathrm{T}=\prod_{P \in\left[\mathcal{S}_{p}(G)\right]} \operatorname{det} \Delta(P) .
$$

The block matrix $\Delta(P)$ is a (square) matrix with rows indexed by the isomorphism classes of indecomposable projective $k \bar{N}_{G}(P)$-modules $E$, and columns indexed by the conjugacy classes of $p^{\prime}$-elements $s$ of $\bar{N}_{G}(P)$. The coefficient $\Delta(P)_{E, s}$ is equal to

$$
\begin{aligned}
\Delta(P)_{E, s} & =t_{P, s}^{G}\left(M_{P, E}\right) \\
& =\operatorname{Brauer} \operatorname{trace}\left(s \mid M_{P, E}[P]\right) \\
& =\Phi_{E}(s)
\end{aligned}
$$

since $M_{P, E}[P] \cong E$, where $\Phi_{E}$ is the Brauer character of the projective $k \bar{N}_{G}(P)$ module $E$.

Similarly,

$$
\operatorname{det} \mathbf{T}^{\prime}=\prod_{P \in\left[\mathcal{S}_{p}(G)\right]} \operatorname{det} \Delta^{\prime}(P)
$$


where the coefficients of the diagonal block $\Delta^{\prime}(P)$ of $\mathrm{T}^{\prime}$ are given by

$$
\Delta^{\prime}(P)_{E, s}=\Phi_{E}\left(s^{-1}\right) \text {. }
$$

2.23. Let $\Sigma(P)$ denote the (square) diagonal matrix indexed by the conjugacy classes of $p^{\prime}$-elements of $\bar{N}_{G}(P)$, with diagonal coefficient $\Sigma(P)_{s, s}$ equal to the inverse of the order of the centralizer $C_{\bar{N}_{G}(P)}(s)$ of $s$ in $\bar{N}_{G}(P)$, and let $U(P)=$ $\Delta(P) \cdot \Sigma(P) \cdot{ }^{t} \Delta^{\prime}(P)$. The coefficient of $U(P)$ indexed by the indecomposable projective $k \bar{N}_{G}(P)$-modules $E$ and $F$ is equal to

$$
\begin{aligned}
U(P)_{E, F} & =\sum_{s \in\left[\bar{N}_{G}(P)_{p^{\prime}}\right]} \Phi_{E}(s) \frac{1}{\left|C_{\bar{N}_{G}(P)}(s)\right|} \Phi_{F}\left(s^{-1}\right) \\
& =\frac{1}{\left|\bar{N}_{G}(P)\right|} \sum_{s \in \bar{N}_{G}(P)_{p^{\prime}}} \Phi_{E}(s) \Phi_{F}\left(s^{-1}\right) \\
& =\langle E, F\rangle_{\bar{N}_{G}(P)} .
\end{aligned}
$$

In other words, the matrix $U(P)$ is equal to the Cartan matrix of the group algebra $k \bar{N}_{G}(P)$. It is then well known (see [6], III.16 or [10]) that

$$
\operatorname{det} U(P)=\prod_{s \in\left[\bar{N}_{G}(P)_{p^{\prime}}\right]}\left|C_{\bar{N}_{G}(P)}(s)\right|_{p},
$$

and it follows that

$$
\begin{aligned}
\operatorname{det} \Delta(P) \operatorname{det}^{t} \Delta^{\prime}(P) & =\frac{\prod_{s \in\left[\bar{N}_{G}(P)_{p^{\prime}}\right]}\left|C_{\bar{N}_{G}(P)}(s)\right|_{p}}{\operatorname{det} \Sigma(P)} \\
& =\prod_{s \in\left[\bar{N}_{G}(P)_{p^{\prime}}\right]}\left|C_{\bar{N}_{G}(P)}(s)\right|_{p} \prod_{s \in\left[\bar{N}_{G}(P)_{p^{\prime}}\right]}\left|C_{\bar{N}_{G}(P)}(s)\right| .
\end{aligned}
$$

Together with (2.20) and (2.21), this gives

$$
\operatorname{det} \mathrm{C}\left(\mu_{k}(G, \mathbf{1})\right)=\prod_{R \in\left[\mathcal{S}_{p}(G)\right]} \prod_{s \in\left[\bar{N}_{G}(R)_{p^{\prime}}\right]}\left(\left|C_{\bar{N}_{G}(R)}(s)\right|_{p}\left|C_{\bar{N}_{G}(R)}(s)\right|\left\langle\left\langle k, F_{R, s}^{G}\right\rangle_{G}\right) .\right.
$$

2.25. Lemma. Let $Q \in \mathcal{S}_{p}(G)$ and $(R, s) \in \mathcal{Q}_{G, p}$. Then

$$
F_{R, s}^{G}[Q]=\sum_{\substack{\left.x \in N_{G}(Q) \backslash G / N_{G}(R, s) \\ Q \unlhd^{x}<R s\right\rangle}} F_{x / Q,{ }^{x} s}^{\bar{N}_{G}(Q)}
$$

(where ${ }^{x} s$ denotes the image in $\bar{N}_{G}\left(Q,{ }^{x} R\right) /{ }^{x} R$ of the $x$-conjugate of $s$ ).

Proof. The Brauer map $W \mapsto W[Q]$ is a ring homomorphism from $p p_{k}(G)$ to $p p_{k}\left(\bar{N}_{G}(Q)\right)$. Thus $F_{R, s}^{G}[Q]$ is an idempotent of $p p_{k}\left(\bar{N}_{G}(Q)\right)$, hence a sum of distinct primitive idempotents $F_{X, u}^{\bar{N}_{G}(Q)}$ associated to some pairs $(X, u) \in \mathcal{Q}_{\bar{N}_{G}(Q), p}$. In other words, $X$ is a $p$-subgroup of $\bar{N}_{G}(Q)$, of the form $Y / Q$, for some $p$-subgroup $Y$ of $G$ such that $Q \unlhd Y$, and $u$ is a $p^{\prime}$-element of $\bar{N}_{\bar{N}_{G}(Q)}(X) \cong N_{G}(Q, Y) / Y$.

The idempotent $F_{X, u}^{\bar{N}_{G}(Q)}$ appears in $F_{R, s}^{G}[Q]$ if and only if the species $t_{X, u}^{\bar{N}_{G}(Q)}$ takes the value 1 when evaluated at $F_{R, s}^{G}[Q]$. But $t_{X, u}^{\bar{N}_{G}(Q)}\left(F_{R, s}^{G}[Q]\right)$ is equal to the 
Brauer trace (see (2.15) ) of $u$ on $F_{R, s}^{G}[Q][Y / Q]=\operatorname{Res}_{N_{G}(Q, Y) / Y}^{N_{G}(Y) / Y} F_{R, s}^{G}[Y]$. In other words,

$$
t_{X, u}^{\bar{N}_{G}(Q)}\left(F_{R, s}^{G}[Q]\right)=t_{Y, u}^{G}\left(F_{R, s}^{G}\right),
$$

and this is equal to 1 if and only if the pair $(Y, u)$ is $G$-conjugate to the pair $(R, s)$. The lemma follows.

2.26. Lemma. Let $(R, s) \in \mathcal{Q}_{G, p}$. Then

$$
\left\langle k, F_{R, s}^{G}\right\rangle_{G}=\left\{\begin{array}{cl}
\frac{\phi(|R|)}{\left|N_{G}(R, s)\right|} & \text { if }\langle s R\rangle \text { is cyclic } \\
0 & \text { otherwise }
\end{array}\right.
$$

where $\phi$ is the Euler totient function.

Proof. Recall from (2.13) that

$$
F_{R, s}^{G}=\frac{1}{|R||s|\left|C_{\bar{N}_{G}(R)}(s)\right|} \sum_{\varphi, L} \tilde{\varphi}\left(s^{-1}\right)|L| \mu(L,\langle s R\rangle) \operatorname{Ind}_{L}^{G} \operatorname{Res}_{L}^{\langle s R\rangle} k_{\varphi}
$$

where $\varphi$ runs through homomorphisms $\langle s\rangle \rightarrow k^{\times}$(i.e. equivalently homomorphisms $\langle s R\rangle \rightarrow k^{\times}$), and $L$ runs through subgroups of $\langle s R\rangle$ such that $L R=\langle s R\rangle$. Now

$$
\left\langle k, \operatorname{Ind}_{L}^{G} \operatorname{Res}_{L}^{\langle s R\rangle} k_{\varphi}\right\rangle_{G}=\left\langle k, k_{\varphi}\right\rangle_{L}
$$

is equal to zero unless the restriction of $\varphi$ to $L$ is trivial, i.e. if $L \leq \operatorname{Ker} \varphi$. Since $R \leq \operatorname{Ker} \varphi$, this implies that $\langle s R\rangle \leq \operatorname{Ker} \varphi$, i.e. that $\varphi$ is trivial. Thus

$$
\left\langle k, F_{R, s}^{G}\right\rangle_{G}=\frac{1}{|R||s|\left|C_{\bar{N}_{G}(R)}(s)\right|} \sum_{L R=\langle s R\rangle}|L| \mu(L,\langle s R\rangle) .
$$

Now a subgroup $L$ of $H=\langle s R\rangle$ is such that $L R=H$ if and only if it contains some conjugate of $s$ in $H$, i.e. if it is of the form $Q\left\langle{ }^{x} s\right\rangle$, for some $x \in R$ and some subgroup $Q$ of $R$ normalized by ${ }^{x} s$. In this case, there are $\left|Q: C_{Q}\left({ }^{x} s\right)\right|$ conjugates of $s$ contained in $L$. Moreover, since $L R=H$ and $L \cap R=O_{p}(H)=Q$, the map $X \mapsto X \cap R$, from the poset ] $L, H$ [ of proper subgroups of $H$ strictly containing $L$, to the poset $] Q, R\left[{ }^{L}=\right] Q, R\left[^{x} s\right.$ of $L$-invariant proper subgroups of $R$ strictly containing $Q$, is an isomorphism: the inverse isomorphism is the map $Y \mapsto Y \cdot L$. Thus $\mu(L,\langle s R\rangle)$ is equal to the value $\mu\left((Q, R)^{x}\right)$ of the Möbius function of the poset of subgroups normalized by ${ }^{x} s$, and

$$
\begin{aligned}
& \left\langle k, F_{R, s}^{G}\right\rangle_{G}=\frac{1}{|R||s|\left|C_{\bar{N}_{G}(R)}(s)\right|} \sum_{x \in R / C_{R}(s)} \sum_{\substack{Q \leq R \\
Q^{x_{s}}=Q}}|Q||s| \mu\left((Q, R)^{x} s\right) /\left|Q: C_{Q}\left({ }^{x} s\right)\right| \\
& =\frac{1}{|R|\left|C_{\bar{N}_{G}(R)}(s)\right|} \sum_{x \in R / C_{R}(s)} \sum_{\substack{Q \leq R \\
Q^{x}=Q}}\left|C_{Q}\left({ }^{x} s\right)\right| \mu\left((Q, R)^{x} s\right) .
\end{aligned}
$$


Now $Q$ is normalized by ${ }^{x} s$ if and only if $Q^{x}$ is normalized by $s$. Moreover, $\mu\left((Q, R)^{x} s\right)=\mu\left(\left(Q^{x}, R\right)^{s}\right)$, and $\left|C_{Q}\left({ }^{x} s\right)\right|=\left|C_{Q^{x}}(s)\right|$. Thus

$$
\begin{aligned}
\left\langle k, F_{R, s}^{G}\right\rangle_{G} & =\frac{1}{|R|\left|C_{\bar{N}_{G}(R)}(s)\right|} \sum_{x \in R / C_{R}(s)} \sum_{\substack{Q \leq R \\
Q^{s}=Q}}\left|C_{Q}(s)\right| \mu\left((Q, R)^{s}\right) \\
& =\frac{1}{\left|C_{R}(s)\right|\left|C_{\bar{N}_{G}(R)}(s)\right|} \sum_{\substack{Q \leq R \\
Q^{s}=Q}}\left|C_{Q}(s)\right| \mu\left((Q, R)^{s}\right) .
\end{aligned}
$$

Now

$$
\begin{aligned}
\sum_{\substack{Q \leq R \\
Q^{s}=Q}}\left|C_{Q}(s)\right| \mu\left((Q, R)^{s}\right) & =\sum_{y \in C_{R}(s)} \sum_{\substack{y \in Q \leq R \\
Q^{s}=Q}} \mu\left((Q, R)^{s}\right) \\
& =\sum_{y \in C_{R}(s)} \delta_{\langle y\rangle, R} \\
& =\left|\left\{y \in C_{R}(s) \mid\langle y\rangle=R\right\}\right| .
\end{aligned}
$$

This is nonzero if and only if $R$ is cyclic and centralized by $s$, i.e. if the group $\langle s R\rangle$ is cyclic. In this case $C_{R}(s)=R$, and

$$
\left\langle k, F_{R, s}^{G}\right\rangle_{G}=\frac{1}{|R|\left|C_{\bar{N}_{G}(R)}(s)\right|} \phi(|R|)=\frac{\phi(|R|)}{\left|N_{G}(R, s)\right|},
$$

since $|R|\left|C_{\bar{N}_{G}(R)}(s)\right|=\left|N_{G}(R, s)\right|$, in view of the exact sequence (2.12). This completes the proof of the lemma.

2.27. Lemma. Let $(R, s) \in \mathcal{Q}_{G, p}$. Then

$$
\left\langle\left\langle k, F_{R, s}^{G}\right\rangle_{G}=\frac{1}{\left|C_{\bar{N}_{G}(R)}(s)\right|} \sum_{x \in R /[\langle s R\rangle, R]} \frac{1}{|x|} .\right.
$$

Proof. By Definition 2.9.

$$
\left\langle\left\langle k, F_{R, s}^{G}\right\rangle\right\rangle_{G}=\sum_{Q \in\left[\mathcal{S}_{p}(G)\right]}\left\langle k, F_{R, s}^{G}[Q]\right\rangle_{\bar{N}_{G}(Q)} .
$$

Hence, by Lemma 2.25 ,

$$
\begin{aligned}
&\left\langle\left\langle k, F_{R, s}^{G}\right\rangle\right\rangle_{G}=\sum_{\substack{Q \in \mathcal{S}_{p}(G)\\
}} \frac{\left|N_{G}(Q)\right|}{|G|} \sum_{\substack{x \in N_{G}(Q) \backslash G / N_{G}(R, s) \\
Q \unlhd}}\left\langle k, F_{x R / Q,,_{s}}^{\bar{N}_{G}(Q)}\right\rangle_{\bar{N}_{G}}(Q) \\
&=\sum_{\substack{Q \in \mathcal{S}_{p}(G) \\
x \in G \\
Q^{x} \unlhd\langle s R\rangle}} \frac{\left|N_{G}(Q)\right|}{|G|} \frac{\left|N_{G}\left(Q^{x}\right) \cap N_{G}(R, s)\right|}{\left|N_{G}(Q)\right|\left|N_{G}(R, s)\right|}\left\langle k, F_{R / Q^{x}, s}^{\bar{N}_{G}\left(Q^{x}\right)}\right\rangle_{\bar{N}_{G}\left(Q^{x}\right)} \\
&=\sum_{\substack{Q \leq R \\
Q \unlhd\langle s R\rangle}} \frac{\left|N_{G}(Q) \cap N_{G}(R, s)\right|}{\left|N_{G}(R, s)\right|}\left\langle k, F_{R / Q, s}^{\bar{N}_{G}(Q)}\right\rangle_{\bar{N}_{G}(Q)} .
\end{aligned}
$$

Now $\left\langle k, F_{R / Q, s}^{\bar{N}_{G}(Q)}\right\rangle_{\bar{N}_{G}(Q)}=0$ by Lemma 2.26, unless $R / Q$ is cyclic and centralized by $s$, i.e. if $R / Q$ is cyclic and $Q$ contains the commutator subgroup $[\langle s R\rangle, R]$, in 
which case it is equal to $\frac{\phi(|R / Q|)}{|M|}$, where $M=N_{\bar{N}_{G}(Q)}(R / Q, s)$. Now the two exact sequences

$$
\begin{aligned}
& \mathbf{1} \rightarrow R \rightarrow N_{G}(Q) \cap N_{G}(R, s) \rightarrow C_{N_{G}(Q, R) / R}(s) \rightarrow \mathbf{1}, \\
& \mathbf{1} \rightarrow R / Q \rightarrow N_{\bar{N}_{G}(Q)}(R / Q, s) \rightarrow C_{N_{G}(Q, R) / R}(s) \rightarrow \mathbf{1}
\end{aligned}
$$

show that

It follows that

$$
\frac{\left|N_{G}(Q) \cap N_{G}(R, s)\right|}{\left|N_{\bar{N}_{G}(Q)}(R / Q, s)\right|}=|Q|
$$

$$
\begin{aligned}
\left\langle\left\langle k, F_{R, s}^{G}\right\rangle\right\rangle_{G} & =\sum_{\substack{[\langle s R\rangle, R] \leq Q \leq R \\
R / Q \text { cyclic }}} \frac{|Q| \phi(|R / Q|)}{\left|N_{G}(R, s)\right|} \\
& =\frac{1}{\left|C_{\bar{N}_{G}(R)}(s)\right|} \sum_{\substack{\langle\langle s R\rangle, R] \leq Q \leq R \\
R / Q \text { cyclic }}} \frac{\phi(|R / Q|)}{|R / Q|} .
\end{aligned}
$$

Now the summation is equal to the summation over all cyclic quotients $X=R / Q$ of the abelian group $A=R /[\langle s R\rangle, R]$ of $\frac{\phi(|X|)}{|X|}$, i.e. by a classical duality argument, to the summation of this quantity over the cyclic subgroups of $A$, i.e. finally to $\sum_{x \in A} \frac{1}{|x|}$. Thus

as was to be shown.

$$
\left\langle\left\langle k, F_{R, s}^{G}\right\rangle\right\rangle_{G}=\frac{1}{\left|C_{\bar{N}_{G}(R)}(s)\right|} \sum_{x \in R /[\langle s R\rangle, R]} \frac{1}{|x|},
$$

2.28. Theorem. Let $G$ be a finite group, let $p$ be a prime number, and let $k$ be a field of characteristic p, big enough to be a splitting field for all the groups $N_{G}(Q) / Q$, for $Q \in \mathcal{S}_{p}(G)$. Then the determinant of the Cartan matrix of the algebra $\mu_{k}(G, \mathbf{1})$ is equal to

$$
\operatorname{det} C\left(\mu_{k}(G, \mathbf{1})\right)=\prod_{R \in\left[\mathcal{S}_{p}(G)\right]} \prod_{s \in\left[\bar{N}_{G}(R)_{p^{\prime}}\right]}\left(\left|C_{\bar{N}_{G}(R)}(s)\right|_{p} \sum_{x \in R /[\langle s R\rangle, R]} \frac{1}{|x|}\right) .
$$

Proof. The Cartan matrix of $\mu_{k}(G, \mathbf{1})$ is independent of the field $k$, as long as it is big enough. So one can assume e.g. that $k$ is algebraically closed, and choose a corresponding $p$-modular system $(K, \mathcal{O}, k)$, where $K$ is also big enough. Then the formula for $\operatorname{det} \mathrm{C}\left(\mu_{k}(G, \mathbf{1})\right)$ follows from equation (2.24) and Lemma 2.27.

2.29. Examples. Recall that $k$ is a (big enough) field of characteristic $p$.

- Let $G$ be a cyclic group of order $p^{n}$. Then

$$
\operatorname{det} C\left(\mu_{k}(G, \mathbf{1})\right)=p^{\left(\begin{array}{c}
n \\
2
\end{array}\right)} \prod_{i=1}^{n}(p+i(p-1)) .
$$

- Let $G$ be an elementary abelian group of order $p^{2}$. Then

$$
\operatorname{det} \mathrm{C}\left(\mu_{k}(G, \mathbf{1})\right)=p(2 p-1)^{p+1}\left(p^{2}+p-1\right) \text {. }
$$




\section{The COHOmological MaCkey Algebra}

The cohomological Mackey algebra $\operatorname{co\mu }_{R}(G)$ of a finite group $G$ over a commutative ring $R$ has also been introduced by Thévenaz and Webb (see [13, Section 16). It is an associative algebra with the property that the category of left $\operatorname{co\mu }_{R}(G)$ modules is equivalent to the category $\operatorname{coMack}_{R}(G)$ of cohomological Mackey functors. The algebra $\operatorname{co\mu }_{R}(G)$ is defined as a quotient of the Mackey algebra $\mu_{R}(G)$.

In the case where $R$ is a field $k$ of characteristic $p$, the cohomological Mackey functors for $G$ over $k$ are projective relative to $p$-subgroups. In other words, the algebra $\operatorname{co\mu }_{k}(G)$ is a quotient of $\mu_{k}(G, \mathbf{1})$.

3.1. Thévenaz and Webb have shown that the projective cohomological Mackey functors for $G$ over $R$ are exactly the fixed point functors $F P_{W}$, where $W$ is a direct summand of some permutation $R G$-module. Moreover, for any $R G$-modules $V$ and $W$

$$
\operatorname{Hom}_{\operatorname{coMack}_{R}(G)}\left(F P_{W}, F P_{V}\right) \cong \operatorname{Hom}_{R G}(W, V) \text {. }
$$

In particular, the indecomposable projective $c o \mu_{k}(G)$-modules are the functors $F P_{W}$, where $W$ is an indecomposable $p$-permutation $k G$-module. Thus, the Cartan matrix of the algebra $\operatorname{co\mu }_{k}(G)$ is the square matrix indexed by the set $\left[\mathcal{P}_{G, p}\right]$, defined by

$$
c_{(P, E),(Q, F)}=\operatorname{dim}_{k} \operatorname{Hom}_{\mathrm{CoMack}_{k}(G)}\left(F P_{M_{P, E}}, F P_{M_{Q, F}}\right)=\left\langle M_{P, E}, M_{Q, F}\right\rangle_{G} .
$$

3.2. Thus, by the same argument already used in Section 2, the Cartan matrix of the algebra $\operatorname{co\mu }_{k}(G)$ is the matrix of the bilinear form $\langle$,$\rangle on p p_{k}(G)$. This extends to a bilinear form on $K \otimes_{\mathbb{Z}} p p_{k}(G)$, and by (2.8), for $(R, s)$ and $\left(R^{\prime}, s^{\prime}\right)$ in $\mathcal{Q}_{G, p}$,

$$
\left\langle F_{R^{\prime}, s^{\prime}}^{G}, F_{R, s}^{G}\right\rangle=\left\{\begin{array}{cl}
\left\langle k, F_{R, s}^{G}\right\rangle_{G} & \text { if }\left(R^{\prime}, s^{\prime}\right)=_{G}\left(R, s^{-1}\right), \\
0 & \text { otherwise. }
\end{array}\right.
$$

By Lemma 2.26, this is nonzero if and only if the group $\langle s R\rangle$ is cyclic.

The argument of paragraph 2.19 shows that the Cartan matrix of $\operatorname{co\mu }_{k}(G)$ can be expressed as

$$
\mathrm{C}\left(\operatorname{co\mu }_{k}(G)\right)=\mathrm{T}^{\prime} \cdot \mathrm{S}^{\prime} \cdot{ }^{t} \mathrm{~T},
$$

where $\mathrm{S}^{\prime}$ is the diagonal matrix indexed by $\left[\mathcal{Q}_{G, p}\right]$ with $(R, s)$-diagonal entry equal to $\left\langle F_{R, s}^{G}, k\right\rangle_{G}$. Since $\mathrm{T}$ and $\mathrm{T}^{\prime}$ are invertible, it follows that the rank of $\mathrm{C}\left(\operatorname{co\mu } \mu_{k}(G)\right)$ is equal to the number of elements $(R, s)$ of $\left[\mathcal{Q}_{G, p}\right]$ such that $\langle s R\rangle$ is cyclic.

3.3. Theorem. Let $G$ be a finite group, let $p$ be a prime number, and let $k$ be a field of characteristic p, big enough to be a splitting field for all the groups $N_{G}(Q) / Q$, for $Q \in \mathcal{S}_{p}(G)$. Then:

(1) The rank of the Cartan matrix of the algebra co $\mu_{k}(G)$ is equal to the number of $G$-conjugacy classes of pairs $(R, s)$, where $R \in \mathcal{S}_{p}(G)$ and $s \in \bar{N}_{G}(R)_{p^{\prime}}$, such that $\langle s R\rangle$ is cyclic. In other words,

$$
\begin{aligned}
\operatorname{rkC}\left(\operatorname{co\mu }_{k}(G)\right) & =\sum_{R \in\left[\mathcal{C}_{p}(G)\right]}\left|N_{G}(R) \backslash C_{G}(R)_{p^{\prime}}\right| \\
& =\sum_{s \in\left[G_{p^{\prime}}\right]} c_{p}\left(C_{G}(s)\right)
\end{aligned}
$$

where $\left[\mathcal{C}_{p}(G)\right]$ denotes a set of representatives of conjugacy classes of cyclic p-subgroups of a group $G$, and $c_{p}(G)$ is the number of such conjugacy 
classes. Moreover, in the first summation, $\left|N_{G}(R) \backslash C_{G}(R)_{p^{\prime}}\right|$ denotes the number of $N_{G}(R)$-conjugacy classes of p-regular elements of $C_{G}(R)$.

(2) The Cartan matrix of the algebra $\operatorname{cop}_{k}(G)$ is nonsingular if and only if the group $G$ is p-nilpotent with cyclic Sylow p-subgroups. In this case, if $G=N \rtimes P$, where $N$ is a $p^{\prime}$-group and $P$ is a cyclic group of order $p^{n}$,

$$
\begin{aligned}
\operatorname{det} C\left(\operatorname{co\mu }_{k}(G)\right) & =\prod_{R \leq P}\left(\frac{\phi(|R|)}{|R|}\right)^{l_{p}\left(\bar{N}_{G}(R)\right)} \operatorname{det} \mathrm{C}\left(k \bar{N}_{G}(R)\right) \\
& =\prod_{R \leq P} \prod_{x \in\left[P \backslash\left[C_{N}(R)\right]\right.}\left(\frac{\phi(|R|)}{|R|}\left|C_{C_{N}(R) \rtimes P / R}(x)\right|_{p}\right),
\end{aligned}
$$

where $l_{p}\left(\bar{N}_{G}(R)\right)$ is the number of p-regular classes of $\bar{N}_{G}(R)$, where $\mathrm{C}\left(k \bar{N}_{G}(R)\right)$ is the Cartan matrix of the group algebra $k \bar{N}_{G}(R)$, and $\left[P \backslash\left[C_{N}(R)\right]\right]$ is a set of representatives of $C_{N}(R) \rtimes P$-conjugacy classes in $C_{N}(R)$.

Proof. The first sentence of Assertion 1 follows from the above arguments, and the second sentence follows by counting the number of pairs $(R, s)$ of $\left[\mathcal{Q}_{G, p}\right]$ such that $\langle s R\rangle$ is cyclic, in two different ways. For Assertion 2, observe that the Cartan matrix $\mathrm{C}\left(\operatorname{co\mu }_{k}(G)\right)$ is nonsingular if and only if for any $(R, s) \in \mathcal{Q}_{G, p}$, the group $\langle s R\rangle$ is cyclic. This amounts to saying that the Sylow $p$-subgroups of $G$ are cyclic, and that whenever $s \in G_{p^{\prime}}$ normalizes a $p$-subgroup $R$, it centralizes it. In other words, the group $N_{G}(R) / C_{G}(R)$ is a $p$-group, for any $R \in \mathcal{S}_{p}(G)$. This is equivalent to saying that $G$ is $p$-nilpotent, by the theorem of Frobenius ([11, Theorem 4.5).

In the case that $G=N \rtimes P$, where $P$ is cyclic of order $p^{n}$, then as in (2.24),

$$
\begin{aligned}
\operatorname{det} \mathrm{C}\left(\operatorname{cop}_{k}(G)\right) & =\operatorname{det} \mathrm{T}^{\prime} \operatorname{det} \mathrm{S}^{\prime} \operatorname{det}^{t} \mathrm{~T} \\
& =\prod_{R \in\left[\mathcal{S}_{p}(G)\right]} \prod_{s \in\left[\bar{N}_{G}(R)_{p^{\prime}}\right]}\left(\left|C_{\bar{N}_{G}(R)}(s)\right|_{p}\left|C_{\bar{N}_{G}(R)}(s)\right|\left\langle k, F_{R, s}^{G}\right\rangle_{G}\right) \\
& =\prod_{R \in\left[\mathcal{S}_{p}(G)\right]}\left(\operatorname{det} \mathrm{C}\left(k \bar{N}_{G}(R)\right) \prod_{s \in\left[\bar{N}_{G}(R)_{p^{\prime}}\right]}\left(\left|C_{\bar{N}_{G}(R)}(s)\right|\left\langle k, F_{R, s}^{G}\right\rangle_{G}\right)\right) .
\end{aligned}
$$

The set $\left[\mathcal{S}_{p}(G)\right]$ can be chosen to be the set of subgroups of $P$. Moreover, by Lemma 2.26, for $R \leq P$ and $s \in \bar{N}_{G}(R)_{p^{\prime}}$,

$$
\left\langle k, F_{R, s}^{G}\right\rangle_{G}=\frac{\phi(|R|)}{\left|N_{G}(R, s)\right|}=\frac{\phi(|R|)}{|R|\left|C_{\bar{N}_{G}(R)}(s)\right|} .
$$

It follows that

$$
\operatorname{det} \mathrm{C}\left(\operatorname{cop}_{k}(G)\right)=\prod_{R \leq P}\left(\frac{\phi(|R|)}{|R|}\right)^{l_{p}\left(\bar{N}_{G}(R)\right)} \operatorname{det} \mathrm{C}\left(k \bar{N}_{G}(R)\right) .
$$

Finally the $p^{\prime}$-elements of the group $\bar{N}_{G}(R) \cong C_{N}(R) \rtimes(P / R)$ are the elements of $C_{N}(R)$. The last formula of the theorem follows. 
3.4. Remark. Thus when it is nonzero, the determinant of the Cartan matrix of $\operatorname{co\mu }_{k}(G)$ is equal to $(p-1)^{n} p^{m}$, for suitable nonnegative intergers $n$ and $m$.

\section{Blocks of COHOMOLOGICAL MaCKey FUnCtors}

4.1. It was shown by Thévenaz and Webb (see [13], Theorem 17.1 and its proof; see also [4)) that the blocks of the algebra $\mu_{k}(G, \mathbf{1})$ and the blocks of the algebra $\operatorname{cop}_{k}(G)$ are in one-to-one correspondence with the blocks of the group algebra $k G$. If $b$ is a block of $k G$, denote by $c o \mu_{k}(b)$ the corresponding block of $\operatorname{co\mu }_{k}(G)$. When $R$ is a $p$-subgroup of $G$, let $\operatorname{Br}_{R}:(k G)^{R} \rightarrow k C_{G}(R)$ denote the Brauer morphism. If moreover $R \unlhd G$, denote by $u \mapsto \bar{u}$ the projection map $k G \rightarrow k(G / R)$. When $A$ is a $k$-algebra, denote by $\operatorname{Irr}_{k}(A)$ the set of isomorphism classes of simple $A$-modules.

This section is devoted to the proof of the following block version of Theorem 3.3 for such blocks of cohomological Mackey functors:

4.2. Theorem. Let $G$ be a finite group, let $p$ be a prime number, and let $k$ be an algebraically closed field of characteristic $p$. Moreover, let $b$ be a block of $k G$. Then:

(1) The rank of the Cartan matrix of the algebra co $\mu_{k}(b)$ is equal to

$$
\begin{aligned}
\operatorname{rkC}\left(c o \mu_{k}(b)\right) & =\sum_{R \in\left[\mathcal{C}_{p}(G)\right]}\left|N_{G}(R) \backslash \operatorname{Irr}_{k}\left(k C_{G}(R) \mathrm{Br}_{R}(b)\right)\right| \\
& =\sum_{(R, c) \in\left[\mathcal{C}_{p}(b)\right]}\left|N_{G}(R, c) \backslash \operatorname{Irr}_{k}\left(k C_{G}(R) c\right)\right|,
\end{aligned}
$$

where $\left[\mathcal{C}_{p}(b)\right]$ is a set of representatives of $G$-conjugacy classes of b-Brauer pairs $(R, c)$ for which $R$ is cyclic.

(2) The Cartan matrix of the block $\operatorname{co\mu }_{k}(b)$ is nonsingular if and only if $b$ is a nilpotent block with cyclic defect groups.

4.3. The Cartan matrix $C$ of $\operatorname{co\mu }_{k}(b)$ is nonsingular if and only if the rows of the decomposition matrix $\mathrm{D}$ are linearly independent (since $\mathrm{C}=\mathrm{D} \cdot{ }^{t} \mathrm{D}$ ): indeed, if a vector $u$ is such that $\mathrm{C} u=0$, then ${ }^{t} u \mathrm{C} u={ }^{t}\left({ }^{t} \mathrm{D} u\right) \cdot\left({ }^{t} \mathrm{D} u\right)=0$, and thus ${ }^{t} \mathrm{D} u=0$. Conversely, if ${ }^{t} \mathrm{D} u=0$, then obviously $\mathrm{C} u=0$.

4.4. The decomposition matrix $\mathrm{D}$ of $\operatorname{co\mu }_{k}(b)$ has rows indexed by the (isomorphism classes of) indecomposable $p$-permutation $k G$-modules in the block $b$, and columns indexed by the (isomorphism classes of) simple $K G$-modules in the block $b$. The coefficient $\mathrm{D}_{W, \chi}$ corresponding to the indecomposable $p$-permutation $k G b$-module $W$ and the simple $K G$-module $\chi$ is equal to the multiplicity of $\chi$ in $K \otimes_{\mathcal{O}} \tilde{W}$, where $\tilde{W}$ is an $\mathcal{O} G$-module lifting $W$ to $\mathcal{O}$ (i.e. such that $k \otimes_{\mathcal{O}} \tilde{W} \cong W$ ). Such an $\mathcal{O} G$-module is unique up to isomorphism, since $W$ is a $p$-permutation module. The character of the module $K \otimes_{\mathcal{O}} \tilde{W}$ will be called the (ordinary) character of $W$.

It follows that the rank of the Cartan matrix of $\operatorname{co\mu }_{k}(b)$ is equal to the dimension of the subspace of $\mathbb{Q} \otimes_{\mathbb{Z}} R_{K}(b)$ generated by characters of $p$-permutation modules in the block $b$.

In particular, the Cartan matrix of $c o \mu_{k}(b)$ is nonsingular if and only if the ordinary characters of the indecomposable $p$-permutation modules in $b$ are linearly independent. 
4.5. Recall (see paragraph 2.15] and [8, Proposition 3.3) that the value of the ordinary character of a $p$-permutation module $W$ on an element $s$ of $G$ is equal to the Brauer trace $\operatorname{Br} \operatorname{Tr}\left(s_{p^{\prime}} \mid W\left[\left\langle s_{p}\right\rangle\right]\right)$, where $s_{p}$ and $s_{p^{\prime}}$ are the $p$-part and $p^{\prime}$-part of $s$, respectively.

4.6. Notation. Let $\mathcal{Z}_{p}(G)$ denote the set of pairs $(P, E)$ consisting of a cyclic p-subgroup $P$ of $G$ and an indecomposable projective $k \bar{C}_{G}(P)$-module $E$, where $\bar{C}_{G}(P)=C_{G}(P) / P$, and let $\left[\mathcal{Z}_{p}(G)\right]$ be a set of representatives of $G$-orbits on $\mathcal{Z}_{p}(G)$.

Let $\mathcal{Z}_{p}(b)$ denote the subset of $\mathcal{Z}_{p}(G)$ consisting of pairs $(P, E)$ such that

$$
\operatorname{Br}_{P}(b) \operatorname{Inf}_{\bar{C}_{G}(P)}^{C_{G}(P)} E=\operatorname{Inf}_{\bar{C}_{G}(P)}^{C_{G}(P)} E .
$$

Moreover, set $\left[\mathcal{Z}_{p}(b)\right]=\mathcal{Z}_{p}(b) \cap\left[\mathcal{Z}_{p}(G)\right]$.

4.7. Lemma. The characters of the modules $\operatorname{Ind}_{C_{G}(P)}^{G} \operatorname{Inf}_{\bar{C}_{G}(P)}^{C_{G}(P)} E$, for $(P, E) \in$ $\left[\mathcal{Z}_{p}(G)\right]$, form a basis of the subspace of $\mathbb{Q} \otimes_{\mathbb{Z}} R_{K}(G)$ generated by the characters of the p-permutation modules.

Proof. Since projective modules are $p$-permutation modules and since induction and inflation preserve this class of modules, the module $L_{P, E}=\operatorname{Ind}_{C_{G}(P)}^{G} \operatorname{Inf}_{\bar{C}_{G}(P)}^{C_{G}(P)} E$, for $(P, E) \in \mathcal{Z}_{p}(G)$, is a $p$-permutation module. Up to isomorphism, this module depends only on the $G$-orbit of $(P, E)$. Moreover, the number of $G$-orbits on the set $\mathcal{Z}_{p}(G)$ is equal to

$$
\left|G \backslash \mathcal{Z}_{p}(G)\right|=\sum_{P \in\left[\mathcal{C}_{p}(G)\right]}\left|N_{G}(P) \backslash \operatorname{Irr}_{k}\left(k C_{G}(P)\right)\right| .
$$

Indeed, the number of isomorphism classes of indecomposable projective $k \bar{C}_{G}(P)$ modules is equal to the number of isomorphism classes of simple $k \bar{C}_{G}(P)$-modules, i.e. to the number of isomorphism classes of simple $k C_{G}(P)$-modules, since $P$ is a normal $p$-subgroup of $C_{G}(P)$.

By Theorem 3.3. it follows that the cardinality of the set $\left[\mathcal{Z}_{p}(G)\right]$ is precisely equal to the rank of the Cartan matrix of the algebra $\operatorname{co\mu }_{k}(G)$, i.e. to the dimension of the subspace of $\mathbb{Q} \otimes_{\mathbb{Z}} R_{K}(G)$ generated by the characters of the $p$-permutation modules. Thus, to prove Lemma 4.7, it is enough to prove that the characters of the modules $L_{P, E}$, for $(P, E) \in\left[\mathcal{Z}_{p}(G)\right]$, are linearly independent.

The character $\chi_{P, E}$ of the module $L_{P, E}$ is equal to $\operatorname{Ind}_{C_{G}(P)}^{G} \operatorname{Inf}_{\bar{C}_{G}(P)}^{C_{G}(P)} \Phi_{E}$, where $\Phi_{E}$ is the character of the module $E$. Suppose that some nontrivial linear combination of these characters is equal to 0 , i.e. that there are integers $n_{P, E} \in \mathbb{Z}$, for $(P, E) \in\left[\mathcal{Z}_{p}(G)\right]$, not all equal to 0 , such that

$$
\sum_{(P, E) \in\left[\mathcal{Z}_{p}(G)\right]} n_{P, E} \chi_{P, E}=0
$$

Let $Q$ be maximal such that there exists $(Q, F) \in\left[\mathcal{Z}_{p}(G)\right]$ with $n_{Q, F} \neq 0$, and let $s$ be a generator of the cyclic group $Q$. By paragraph 4.5 for any $t \in C_{G}(s)_{p^{\prime}}$, the value of the linear combination (4.8) at the element st is equal to

$$
0=\sum_{(P, E) \in\left[\mathcal{Z}_{p}(G)\right]} n_{P, E} \operatorname{Br} \operatorname{Tr}\left(t \mid L_{P, E}[Q]\right)
$$


But $L_{P, E}[Q]=0$, unless some conjugate of $Q$ is contained in $P$. By maximality of $Q$, it follows that

$$
0=\sum_{E} n_{Q, E} \operatorname{Br} \operatorname{Tr}\left(t \mid L_{Q, E}[Q]\right)
$$

where $E$ runs through a set $[\mathcal{P}]$ of indecomposable projective $k \bar{C}_{G}(Q)$-modules, up to isomorphism and conjugation by $N_{G}(Q)$. Since moreover

$$
L_{Q, E}[Q] \cong \operatorname{Ind}_{C_{G}(Q)}^{N_{G}(Q)} \operatorname{Inf}_{\bar{C}_{G}(Q)}^{C_{G}(Q)} E
$$

it follows that for any $t \in C_{G}(Q)_{p^{\prime}}$,

$$
\sum_{E \in[\mathcal{P}]} n_{Q, E}\left(\operatorname{Ind}_{C_{G}(Q)}^{N_{G}(Q)} \operatorname{Inf}_{\bar{C}_{G}(Q)}^{C_{G}(Q)} \Phi_{E}\right)(t)=0
$$

This is also equal to

$$
\begin{aligned}
\sum_{E \in[\mathcal{P}]} n_{Q, E}\left(\operatorname{Res}_{C_{G}(Q)}^{N_{G}(Q)} \operatorname{Ind}_{C_{G}(Q)}^{N_{G}(Q)} \operatorname{Inf}_{\bar{C}_{G}(Q)}^{C_{G}(Q)} \Phi_{E}\right)(t) & =\sum_{\substack{E \in[\mathcal{P}] \\
x \in N_{G}(Q) / C_{G}(Q)}} n_{Q, E} \Phi_{E}(\bar{x} \bar{t}) \\
& =\sum_{\substack{E \in[\mathcal{P}] \\
x \in N_{G}(Q) / C_{G}(Q)}} n_{Q, E} \Phi_{E^{x}}(\bar{t}),
\end{aligned}
$$

where $\bar{t}$ is the image of $t$ in $\bar{C}_{G}(Q)$, and $E^{x}$ is the image of $E$ by conjugation by $x \in N_{G}(Q)$.

Since the map $t \mapsto \bar{t}$ is a surjection from $C_{G}(Q)_{p^{\prime}}$ to $\bar{C}_{G}(Q)_{p^{\prime}}$, it follows that the modular character

$$
\sum_{\substack{E \in[\mathcal{P}] \\ x \in N_{G}(Q) / C_{G}(Q)}} n_{Q, E} \Phi_{E^{x}}
$$

of $\bar{C}_{G}(Q)$ is equal to zero. But the set of modules $E^{x}$, for $E \in[\mathcal{P}]$ and $x \in$ $N_{G}(Q) / C_{G}(Q)$, is exactly the set of projective indecomposable $k \bar{C}_{G}(Q)$-modules, up to isomorphism. Thus

$$
\sum_{F \in \mathcal{Q}} n_{Q, F} m_{F} \Phi_{F}=0
$$

where $\mathcal{Q}$ is a set of representatives of isomorphism classes of indecomposable projective $k \bar{C}_{G}(Q)$-modules, where $n_{Q, F}$ is defined as $n_{Q, E}$ if $(Q, E) \in[\mathcal{P}]$ and if there exists $x \in N_{G}(Q)$ such that $E^{x} \cong F$, and where $m_{F}$ is the number of elements $x \in N_{G}(Q) / C_{G}(Q)$ such that $F^{x} \cong F$.

Now the characters $\Phi_{F}$, for $F \in \mathcal{Q}$, are linearly independent, and it follows that $n_{Q, F}=0$ for any $F$. This contradicts the definition of $Q$, and completes the proof of Lemma 4.7.

4.9. Notation. Let $P \in \mathcal{S}_{p}(G)$, and let $E$ be any projective $k \bar{N}_{G}(P)$-module. Then $E$ splits as a direct sum $\bigoplus_{i \in I} E_{i}$ of indecomposable $k \bar{N}_{G}(P)$-modules $E_{i}$. In this situation, set $M_{P, E}=\bigoplus_{i \in I} M_{P, E_{i}}$. 
4.10. Lemma. The characters of the modules $M_{P, \operatorname{Ind}_{\bar{C}_{G}(P)}^{\bar{N}_{G}(P)}}$, for $(P, E) \in\left[\mathcal{Z}_{p}(b)\right]$, form a basis of the subspace of $\mathbb{Q} \otimes_{\mathbb{Z}} R_{K}(b)$ generated by the characters of the $p$ permutation modules in the block $b$.

Proof. Let $(P, E) \in \mathcal{Z}_{p}(b)$. The module $M=\operatorname{Ind}_{\bar{C}_{G}(P)}^{\bar{N}_{G}(P)} E$ splits as a direct sum $\bigoplus_{i \in I} E_{i}$ of indecomposable $k \bar{N}_{G}(P)$-modules $E_{i}$. Since $\operatorname{Br}_{P}(b)$ is $N_{G}(P)$-invariant and belongs to $k C_{G}(P)$, it follows that $\overline{\operatorname{Br}_{P}(b)}$ acts as the identity on $M$, hence on every direct summand $E_{i}$. Recall that the indecomposable module $M_{P, E_{i}}$ belongs to the block $b$ if and only if $\overline{\operatorname{Br}_{P}(b)} E_{i}=E_{i}$ (see 2, Corollary 6.3.2). Hence all the indecomposable $k G$-modules $M_{P, E_{i}}$ are in the block $b$, and their direct sum $M_{P, \operatorname{Ind} \overline{\bar{C}}_{G}(P)}$ is also in $b$.

To prove Lemma 4.10, it suffices to observe that the sets $\mathcal{Z}_{p}(b)$, when $b$ runs through the blocks of $k G$, form a partition of $\mathcal{Z}_{p}(G)$, and to prove that the characters of the modules $M_{P, \operatorname{Ind}_{\bar{C}_{G}(P)}^{\bar{N}_{G}(P)}}$, for $(P, E) \in\left[\mathcal{Z}_{p}(G)\right]$, form a basis of the subspace of $\mathbb{Q} \otimes_{\mathbb{Z}} R_{K}(G)$ generated by the characters of the $p$-permutation modules.

For this, observe that

$$
\operatorname{Ind}_{C_{G}(P)}^{G} \operatorname{Inf}_{\bar{C}_{G}(P)}^{C_{G}(P)} E \cong \operatorname{Ind}_{N_{G}(P)}^{G} \operatorname{Inf}_{\bar{N}_{G}(P)}^{N_{G}(P)} \operatorname{Ind}_{\bar{C}_{G}(P)}^{\bar{N}_{G}(P)} E
$$

is a direct sum of $M_{P, \operatorname{Ind}_{\bar{C}_{G}(P)}^{\bar{N}_{G}(P)}}$ and of indecomposable modules with vertex strictly contained in $P$ up to conjugation. This yields a triangular transition matrix, with nonzero diagonal coefficients, and such a matrix changes a basis to another basis.

4.11. The first equality in Assertion 1 of Theorem 4.2 follows trivially from Lemma 4.10. The second equality follows from the fact that, for any $b$-Brauer pair $(R, c)$,

$$
\left|N_{G}(R) \backslash \operatorname{Irr}_{k}\left(k C_{G}(R) \operatorname{Tr}_{N_{G}(R, c)}^{N_{G}(R)} c\right)\right|=\left|N_{G}(R, c) \backslash \operatorname{Irr}_{k}\left(k C_{G}(R) c\right)\right| .
$$

This is because the algebra $k C_{G}(R) \operatorname{Tr}_{N_{G}(R, c)}^{N_{G}(R)} c$ is isomorphic to the direct sum of the block algebras $k C_{G}(R)^{x} c$, for $x \in\left[N_{G}(R) / N_{G}(R, c)\right]$, which are transitively permuted by $N_{G}(R)$. Moreover the stabilizer in $N_{G}(R)$ of $k C_{G}(R) c$ is equal to $N_{G}(R, c)$.

4.12. For any $p$-subgroup $R$ of $G$, induction from $C_{G}(R)$ to $N_{G}(R)$ induces an inequality

$$
\left|N_{G}(R) \backslash \operatorname{Irr}_{k}\left(k C_{G}(R) \operatorname{Br}_{R}(b)\right)\right| \leq\left|\operatorname{Irr}_{k}\left(k N_{G}(R) \operatorname{Br}_{R}(b)\right)\right| .
$$

Indeed, the left hand side is equal to the rank of the group

$$
P_{k}\left(k C_{G}(R) \operatorname{Br}_{R}(b)\right)_{N_{G}(R)}
$$

of $N_{G}(R)$-coinvariants on the group of projective $k C_{G}(R) \mathrm{Br}_{R}(b)$-modules. It is easy to see that $\operatorname{Ind}_{C_{G}(R)}^{N_{G}(R)}$ induces an injective map from this group to the group $P_{k}\left(k N_{G}(R) \mathrm{Br}_{R}(b)\right)$ of projective $k N_{G}(R) \mathrm{Br}_{R}(b)$-modules, and the rank of this group is equal to the right hand side of (4.13). 
The Cartan matrix of $\operatorname{co\mu }_{k}(b)$ is nonsingular if and only if

$$
\sum_{R \in\left[\mathcal{C}_{p}(G)\right]}\left|N_{G}(R) \backslash \operatorname{Irr}_{k}\left(k C_{G}(R) \operatorname{Br}_{R}(b)\right)\right|=\sum_{R \in\left[\mathcal{S}_{p}(G)\right]}\left|\operatorname{Irr}_{k}\left(k N_{G}(R) \operatorname{Br}_{R}(b)\right)\right| .
$$

Indeed, the left hand side is the rank of the Cartan matrix, and the right hand side is the size of this matrix, i.e. the number of indecomposable $p$-permutation modules in the block $b$, up to isomorphism.

By inequality (4.13), this is in turn equivalent to the following equality, for any $R \in \mathcal{S}_{p}(G):$

$$
\left|\operatorname{Irr}_{k}\left(k N_{G}(R) \operatorname{Br}_{R}(b)\right)\right|=\left\{\begin{array}{cl}
\left|N_{G}(R) \backslash \operatorname{Irr}_{k}\left(k C_{G}(R) \operatorname{Br}_{R}(b)\right)\right| & \text { if } R \text { is cyclic, } \\
0 & \text { otherwise. }
\end{array}\right.
$$

4.14. Hence if the Cartan matrix of $\operatorname{co\mu }_{k}(b)$ is nonsingular, and if $b$ has defect $D$, then in particular $\operatorname{Br}_{D}(b) \neq 0$, and $\left|\operatorname{Irr}_{k}\left(k N_{G}(D) \mathrm{Br}_{D}(b)\right)\right| \neq 0$, so $D$ is cyclic. Let $(D, c)$ be a maximal $b$-Brauer pair. Then $\operatorname{Br}_{D}(b)=\operatorname{Tr}_{N_{G}(D, c)}^{N_{G}(D)} c$, and the algebra $k N_{G}(D) \mathrm{Br}_{D}(b)$ is isomorphic to a matrix algebra over $k N_{G}(D, c) c$. In particular

$$
\left|\operatorname{Irr}_{k}\left(k N_{G}(D) \operatorname{Br}_{D}(b)\right)\right|=\left|\operatorname{Irr}_{k}\left(k N_{G}(D, c) c\right)\right| .
$$

Moreover $\mathrm{Br}_{D}(b)$ splits as a sum of blocks of $C_{G}(D)$, which are the distinct $N_{G}(D)$ conjugates of $c$. Hence, the orbits of $N_{G}(D)$ on $\operatorname{Irr}_{k}\left(k C_{G}(D) \mathrm{Br}_{D}(b)\right)$ are in oneto-one correspondence with the orbits of $N_{G}(D, c)$ on $\operatorname{Irr}_{k}\left(k C_{G}(D) c\right)$. It follows that

$$
\left|N_{G}(D, c) \backslash \operatorname{Irr}_{k}\left(k C_{G}(D) c\right)\right|=\left|\operatorname{Irr}_{k}\left(k N_{G}(D, c) c\right)\right| .
$$

Now the group $N_{G}(D, c) / C_{G}(D)$ is the inertial quotient of $b$. It is a cyclic group of order $e$ dividing $p-1$. The block $c$ is a nilpotent block of $C_{G}(D)$, so in particular there is a unique simple $k C_{G}(D) c$-module $S$, which is invariant by $N_{G}(D, c)$. This simple module can be extended to a simple $k N_{G}(D, c) c$-module in $e$ different ways by the following argument (see 2], proof of Proposition 6.5.4): let $g$ be a generator of the group $N_{G}(Q) / C_{G}(Q)$, and $\theta: S \rightarrow S^{g}$ an isomorphism of $k C_{G}(Q)$-modules. Then the map $g^{e}(s) \mapsto \theta^{e}(s)$ is a $k C_{G}(Q)$ automorphism of $S$, because $g^{e} \in C_{G}(Q)$. As $k$ is algebraically closed, there is a scalar $\mu \in k$ such that $g^{e}(s)=\mu \theta^{e}(s)$, for any $s \in S$. Moreover, there are $e$ distinct elements $\lambda \in k$ such that $\lambda^{e}=\mu$. For each such $\lambda$, one can let $g$ act on $S$ by $\lambda \theta$, and this gives $e$ mutually nonisomorphic extensions of $S$ to a simple $k N_{G}(Q, c)$-module, which are all in the block $c$ since $c \in k C_{G}(Q)$.

These modules are not isomorphic to each other (since their restrictions to $\langle g\rangle$ are not). Hence $e=1$ by (4.15), since $\left|\operatorname{Irr}_{k}\left(k C_{G}(D) c\right)\right|=1$, and this is equivalent to saying that $b$ is nilpotent, since $b$ has cyclic defect.

4.16. Conversely, if $b$ is a nilpotent block with a cyclic defect group $D$, then for each $R \in \mathcal{S}_{p}(G)$, either $\operatorname{Br}_{R}(b)=0$ if $R$ is not contained in $D$ up to $G$ conjugation, or $\mathrm{Br}_{R}(b)$ is a sum $\sum_{i \in I} b_{i}$ of blocks $b_{i}$ of $N_{G}(R)$ if $R$ is contained in $D$ up to conjugation, and in that case $R$ is cyclic since $D$ is. Each of the blocks $b_{i}$ is equal to $\operatorname{Tr}_{N_{G}\left(R, c_{i}\right)}^{N_{G}(R)} c_{i}$, where $\left(R, c_{i}\right)$ is some $b$-Brauer pair. In particular

$$
\begin{aligned}
\left|\operatorname{Irr}_{k}\left(k N_{G}(R) \operatorname{Br}_{R}(b)\right)\right| & =\sum_{i \in I}\left|\operatorname{Irr}_{k}\left(k N_{G}(R) \operatorname{Br}_{R}\left(b_{i}\right)\right)\right| \\
& =\sum_{i \in I}\left|\operatorname{Irr}_{k}\left(k N_{G}\left(R, c_{i}\right) c_{i}\right)\right| .
\end{aligned}
$$


Since the block $b$ is nilpotent, all the groups $N_{G}\left(R, c_{i}\right) / C_{G}(R)$ are $p$-groups, so $\left|\operatorname{Irr}_{k}\left(k N_{G}\left(R, c_{i}\right) c_{i}\right)\right|=\left|N_{G}\left(R, c_{i}\right) \backslash \operatorname{Irr}_{k}\left(k C_{G}(R) c_{i}\right)\right|$, and this is equal to 1 since $c_{i}$ is a nilpotent block of $C_{G}(R)$ by Theorem 1.2 of 9 . It follows that

$$
\left|\operatorname{Irr}_{k}\left(k N_{G}(R) \operatorname{Br}_{R}(b)\right)\right|=|I|=\left|N_{G}(R) \backslash \operatorname{Irr}_{k}\left(k C_{G}(R) \operatorname{Br}_{R}(b)\right)\right| .
$$

This implies that the Cartan matrix of $\operatorname{co\mu }_{k}(b)$ is nonsingular and completes the proof of Theorem 4.2 ,

\section{ACKNOWLEDGMENT}

The author wishes to thank Jacques Thévenaz for very fruitful discussions and friendly collaboration on all these questions at the EPFL in June 2009.

\section{REFERENCES}

[1] D. Benson. Modular representation theory: New trends and methods, volume 1081 of Lecture Notes in Mathematics. Springer, 1984. MR765858 (86g:20013)

[2] D. Benson. Representations and cohomology I, volume 30 of Cambridge studies in advanced mathematics. Cambridge University Press, 1991. MR.1110581 (92m:20005)

[3] S. Bouc. Résolutions de foncteurs de Mackey. In Group representations: Cohomology, Group Actions and Topology, volume 63, pages 31-83. AMS Proceedings of Symposia in Pure Mathematics, 1998. MR1603131 (99b:20015)

[4] S. Bouc. The $p$-blocks of the Mackey algebra. Algebras and Representation Theory, 6:515-543, 2003. MR2026725 (2004k:20024)

[5] S. Bouc and J. Thévenaz. The primitive idempotents of the p-permutation ring. Journal of Algebra, 323(10):2905-2915, 2010. MR2609181

[6] R. Brauer and C. Nesbitt. On the modular characters of groups. Ann. of Math. (2), 42(2):556590, 1941. MR0004042 (2:309c)

[7] M. Broué. On Scott modules and p-permutation modules: an approach through the Brauer morphism. Proc. Amer. Math. Soc., 93(3):401-408, March 1985. MR773988 (86d:20010)

[8] M. Broué. Rickard equivalences and block theory, volume 211 of London. Math. Soc. Lecture Note, pages 58-79. Cambridge Univ. Press, 1995. MR1342782 (96d:20011)

[9] M. Broué and L. Puig. A Frobenius theorem for blocks. Invent. Math., 56:117-128, 1980. MR.558864 (81d:20011)

[10] W. Feit. The representation theory of finite groups. North-Holland Publishing Company, 1982. MR661045 (83g:20001)

[11] D. Gorenstein. Finite groups. Harper \& Row, 1968. MR0231903 (38:229)

[12] M. Nicollerat. Foncteurs de Mackey projectifs. Ph.D. thesis, Ecole Polytechnique Fédérale de Lausanne, 2008.

[13] J. Thévenaz and P. Webb. The structure of Mackey functors. Trans. Amer. Math. Soc., 347(6):1865-1961, June 1995. MR1261590 (95i:20018)

LAMFA - CNRS UMR 6140, Université de Picardie Jules Verne, 33, Rue St Leu, 80039 Amiens, France

E-mail address: serge.bouc@u-picardie.fr 